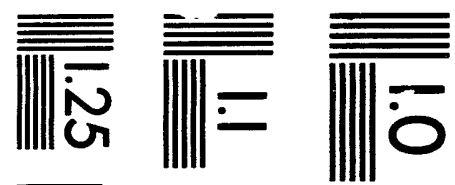

$$
\begin{aligned}
& \text { 菏 }
\end{aligned}
$$

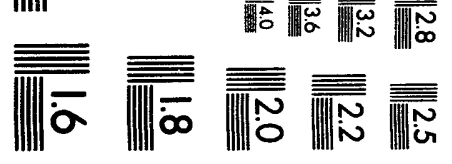



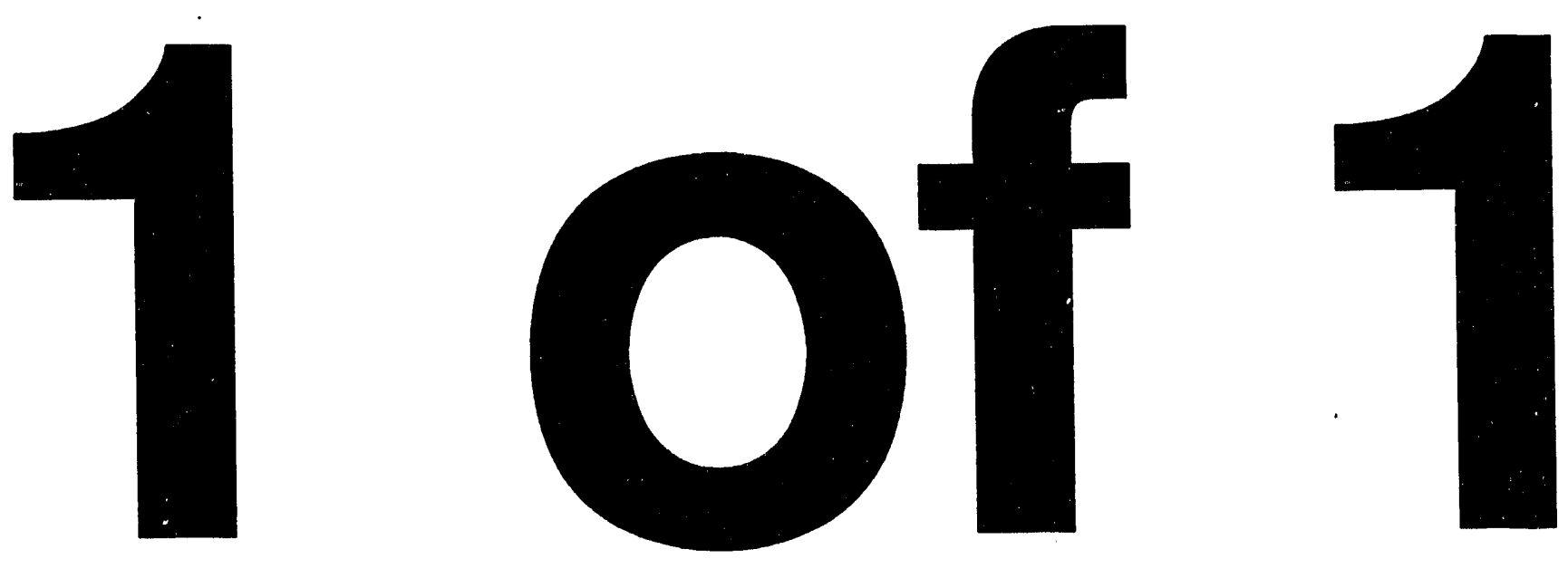


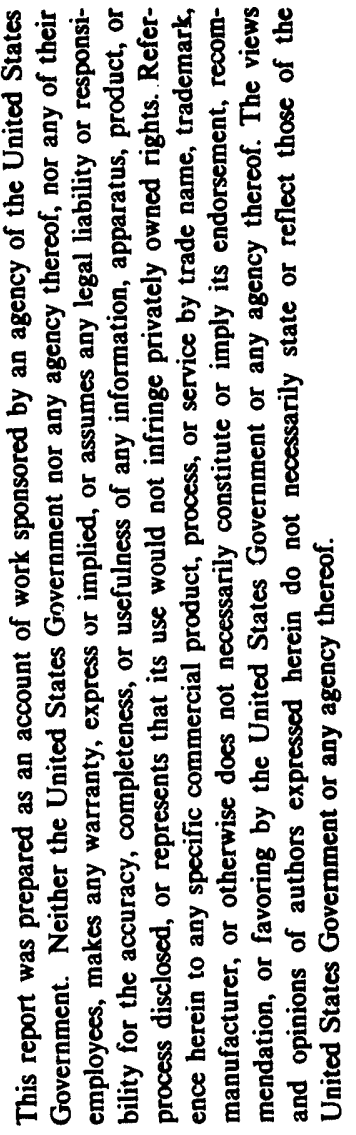

YUVAL KLUGER, T-8, MS B285

LOS ALAMOS NATIONAL LABORATORY

LOS ALAMOS, NM 87545

PROCEEDINGS OF NATO ADVANCED STUDY INSTITUTE

" HOT AND DENSE NUCLEAR MATTER "

HELD IN BODRUM, TURKEY, 9/26-10/9/93.

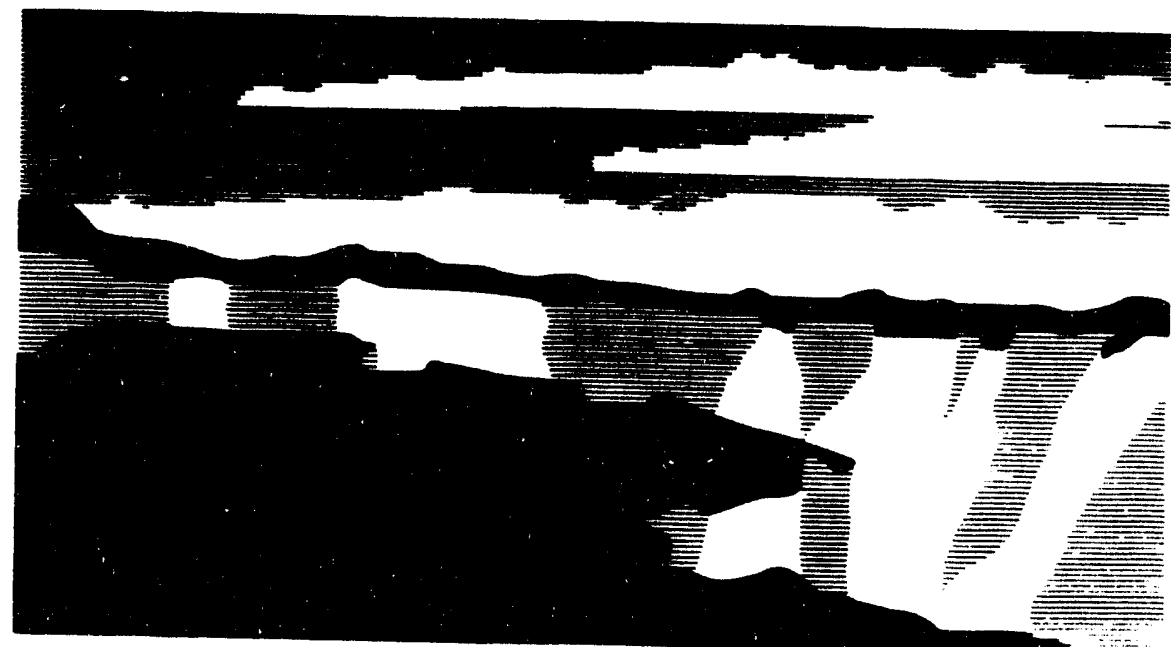

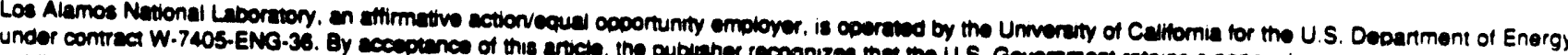

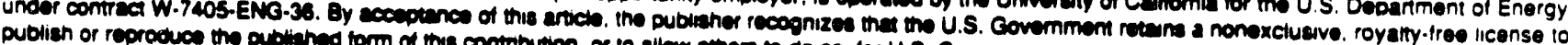

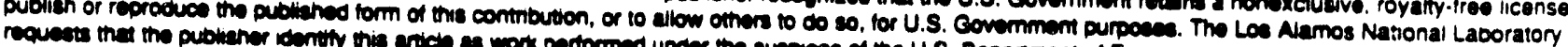




\title{
BACK-REACTION BEYOND THE MEAN FIELD APPROXIMATION
}

\author{
Yuval Kluger \\ Theoretical Division \\ Los Alamos National Laboratory \\ Los Alamos, New Mexico 87545 USA
}

\begin{abstract}
A method for solving an initial value problem of a closed system consisting of an electromagnetic mean field and its quantum fluctuations coupled to fermions is presented. By tailoring the large $N_{f}$ expansion method to the Schwinger-Keldysh closed time path (CTP) formulation of the quantum effective action, causality of the resulting equations of motion is ensured, and a systematic energy conser wing and gauge invariant expansion about the electromagnetic mean field in powers of $1 / N_{f}$ is developed. The resulting equations may be used to study the quantum nonequilibrium effects of pair creation in strong electric fields and the sestering aricl transport processes of a relativistic $e^{+} e^{-}$plasma. Using the Bjork $\mu$ z satz sf hoost invariance initial conditions in which the initial electric mean fir de depencs $c$. the proper time only, we show numerical results for the case in wh thes spansion is truncated in the lowest order, and compare them with those a p tecorsenological transport equation.
\end{abstract}

\section{INTRODUCTION}

In the last few years it has become clear that there are many situations of direct physical interest involving time-dependent dynamical evolution, where a perturbative scattering formalism is wholly inadequate. This is the case for strong field electrodynamics in astrophysical plasmas, in nuclear collisions of heavy ions where a phase 
transition to a quark siuon plasma is expected to take place and in the hot dense early universe. These problems require detailed knowledge of the dynamical time evolution of the field configurations and energy flow.

An understanding of the dynamics of the quark-gluon plasma following its formation in a heavy ion collision is especially important for the theoretical interpretation of the data that will be produced by RHIC experiments within a few years. One would like to know how the plasma is produced, how efficiently it thermalizes and on what time scale, how it evolves and what are the special signatures (in terms of flow of energy, production of particles, etc.) of this new state of matter.

In the early stages of quark-gluon plasma evolution, phenomenological approaches based on kinetic theory (and subsequently on hydrodynamics) are frequently used ${ }^{1-5}$. The event generators which supply a source term for the kinetic equations in the present models are constructed in the framework of a perturbation theory ${ }^{5-6}$. But, in the range of RHIC energies non perturbative effects like the formation of coherent color-electric fields may play an essential role together with the perturbative quantum fluctuations [see the lecture by Gyulassy at this school]. Moreover, effects like off-shell propagation are not taken into account in these studies [see the lectures by $\mathrm{C}$. Greiner and Neise at this school].

The kinetic approach is an intuitive and tractable one. But, it is not known a priori in such a complex system, governed by strong interactions, if it is applicable at all. In order to verify which (if any) of the assumptions of the various kinetic models are valid, it will be useful to investigate whether t'ie inclusion of these various effects in a kinetic model is appropriate for describing the physics. This can be done in three stages: Firstly, by deriving the equations of motion for the mean fields and their quantum fluctuations using the close-time-path method ${ }^{7-9}$ and using a systematic approximation (such as the large $N_{f}$ expansion ${ }^{10-1 j}$ ) to the Schwinger-Dyson equations of the full field theory. Secondly, by extracting the transport equations by using approximations like the gradient expansion ${ }^{14}$ of Green's functions while keeping consistency with the field theory expansion method in use. Thirdly, by comparing the simulations of the field-theory with those of the correspending transport equation ${ }^{15}$. If the transport equation does not reproduce similar results to those of the field theory, one must rely on the full field theory approach which is much more complicated.

A practical starting point for this task would be to deal with a simpler theory like $\mathrm{QED}^{16-19}$, instead of QCD.

I begin with an introduction of the effective action techniques, which are used in the derivation of the effective equations of motion in $\mathrm{QED}^{20}$ (the first stage). I briefly review the functional method of the effective action in the large $N$ expansion 
for QED, then the basics of the Schwinger-Keldysh real time CTP formalism and then I bring the two ingredients together and present ihe effective action and causal equations of motion for electrodynamics.

Finally, I demonstrate the numerical results ${ }^{21}$ which were obtained by applying the above methods in the lowest order of the $1 / N_{f}$ expansion for the case when the initial electric mean field is taken to be a function only of the fluid proper time $\tau=\sqrt{t^{2}-z^{2}}$. These results are compared with those obtained by solving a relativistic transport theory with a modified proper time dependent Schwinger source term $^{22}$ using boost-invariant coordinates ${ }^{1-4}$. In both cases, these initial conditions kinematically guarantee a flat particle rapidity distribution. The motivation to choose these initial conditions is the fact that in heavy-ion collisions one is dealing with a situation in which particle production in the central rapidity region can be modeled as an inside-outside cascade which is symmetric under longitudinal boosts, and thus produces a plateau in the particle rapidity distributions $\mathbf{s}^{23}$.

\section{THE LARGE N EXPANSION}

The large $N_{f}$ expansion is a perturbation functional method which consist of $N_{f}$ identical fields. For QED the $N_{f}$ copies are charged fermions fields (flavors). This method provides a convenient way of parameterizing the separation of the quantum fields into mean fields and their fluctuations and a systematic approximation scheme to the Schwinger-Dyson equations of the full field theory. While the large $N_{f}$ method is not the only technique possible, it is a gauge and renormalization group invariant expansion which arranges the Feynman perturbation series for scattering diagrams in a way that automatically includes self-energy corrections of scattering to the same order. Only fields which scale like $N_{f}$ to a positive power for large $N_{f}$ can be considered strong mean fields in this approach. For such strong fields the leading order approximation is equivalent to the mean field approximation. The Schwinger mechanism for pair production is already realized in the leading order. In the next to leading order in the expansion in powers of $1 / N_{f}$ ihe effects of collisional and radiation processes back-react on the mean fields and appear for the first time. These processes are essential mechanisms for driving the system to equilibrium.

For QED with $N$ identical charged fermion fields the Lagrangian is

$$
L=-\sum_{i=1}^{N} \int d^{4} x \bar{\psi}_{i} G^{-1}[A] \psi_{i}-\frac{N}{4 e^{2}} \int d^{4} x F_{\mu \nu} F^{\mu \nu}
$$


where $G^{-1}[A]$ is the inverse fermion propagtor defined as

$$
G^{-1}[A]=i \frac{\gamma^{\mu}}{2}\left(\vec{\partial}_{\mu}-\overleftarrow{\partial}_{\mu}\right)+\gamma^{\mu} A_{\mu}+i m
$$

Introducing external sources for the gauge potential and Dirac fields, we define the generating functional

$$
\begin{aligned}
Z[J, \eta, \bar{\eta}] \equiv e^{i N W[J, \eta, \bar{\eta}]} \equiv \int & {\left[\mathcal{D} A_{\mu}\right]^{\prime} \prod_{i=1}^{N} \int\left[\mathcal{D} \psi_{i}\right]\left[\mathcal{D} \bar{\psi}_{i}\right] \exp \left\{i \int d^{4} x L[A, \psi, \bar{\psi}]\right\} } \\
& \times \exp \{i N J \circ A+i \bar{\eta} \circ \psi+i \bar{\psi} \circ \eta\}
\end{aligned}
$$

where the prime on the gauge field integration measure denotes that we should integrate only over distinct gauge invariant configurations (or equivalently, fix the gauge), and where the symbol $\circ$ will denote summation over internal indices and integration over continuous spacetime coordinates in the quantities on either side of it (the de Witt summation convention).

Performing the Gaussian integration over the anti-commuting Dirac fields, and rescaling the Grassman valued sources $\eta \rightarrow \sqrt{N} \eta$ so that we can drop the sums over $i=1, \ldots, N$, we obtain

$$
\begin{aligned}
Z[J, \eta, \bar{\eta}]= & \int\left[\mathcal{D} A_{\mu}\right]^{\prime} \exp \left\{i N \int_{j} d^{4} x \frac{1}{2 e^{2}} A_{\mu}\left(g^{\mu \nu} \square-\partial^{\mu} \partial^{\nu}\right) A_{\nu}\right\} \\
& \times \exp \left\{N \operatorname{Tr} \ln G^{-1}+i N \bar{\eta} \circ G[A] \circ \eta+i N J \circ A\right\},
\end{aligned}
$$

where $T r$ denotes summation over internal indices and integration over spacetime coordinate. We have defined the sources and coupling with the correct powers of $N$ to justify performing the remaining functional integration over the electromagnetic potential by the stationary phase method $^{13}$. Varying the argument of the exponent in eq. (4) with respect to $A$ the stationary phase value $A_{\mu}^{s}[J, \eta, \bar{\eta}]$ satisfies

$$
\frac{1}{e^{2}}\left(g^{\mu \nu} \square-\partial^{\mu} \partial^{\nu}\right) A_{\nu}^{s}(x)-i \operatorname{tr} G\left[A^{s}\right](x, x) \gamma^{\mu}-\bar{\eta} \circ G\left[A^{s}\right](, x) \gamma^{\mu} G\left[A^{s}\right](x,) \circ \eta+J^{a}(x)=0,
$$

where $t r$ denotes the Dirac matrix trace only (without integration over spacetime coordinates). The second derivative of the exponent in (4) at its stationary point is $-i N \operatorname{Tr} D^{-1}$, where

$$
\begin{aligned}
D^{-1}\left[A^{s}\right](x, y)^{\mu \nu}= & -\frac{1}{e^{2}}\left(g^{\mu \nu} \square-\partial^{\mu} \partial^{\nu}\right) \delta^{4}(x, y)-i \operatorname{tr}\left[G(y, x) \gamma^{\mu} G(x, y) \gamma^{\nu}\right]_{A=A^{*}}(6) \\
& -2 \operatorname{tr}\left[\bar{\eta} \circ G(, x) \gamma^{\mu} G(x, y) \gamma^{\nu} G(y,) \circ \eta\right]_{A=A^{\bullet}},
\end{aligned}
$$

and where symmetrization with respect to interchange of the pair of spacetime labels $x, \mu$ with $y, \nu$ is understood. Thus, the result of the stationary phase evaluation of 
(3) and (4) is

$$
\begin{aligned}
W[J, \eta, \bar{\eta}] \equiv & W^{(0)}+\frac{1}{N} W^{(1)}+\frac{1}{N^{2}} W^{(2)}+\ldots \\
= & -A^{s} \circ d^{-1} \circ A^{s}+J \circ A^{s}+\bar{\eta} \circ G\left[A^{s}\right] \circ \eta \\
& \quad-i \operatorname{Tr} \ln G^{-1}\left[A^{s}\right]+\frac{i}{2 N} \operatorname{Tr} \ln D^{-1}\left[A^{s}\right]+\mathcal{O}\left(\frac{1}{N^{2}}\right)
\end{aligned}
$$

where

$$
d^{-1}(x, y)^{\mu \nu} \equiv-\frac{1}{e^{2}}\left(g^{\mu \nu} \square-\partial^{\mu} \partial^{\nu}\right) \delta^{4}(x, y)
$$

is the differential operator from the classical action.

The c-number fields are given by the variations,

$$
A_{c}(x) \equiv \frac{\delta W}{\delta J(x)} \quad ; \quad \psi_{c}(x) \equiv \frac{\delta W}{\delta \bar{\eta}(x)} \quad ; \bar{\psi}_{c}(x) \equiv \frac{\delta W}{\delta \eta(x)} \quad .
$$

Note that the c-number field $A_{c}$ differs from the stationary phase point of the Gaussian integral at order $1 / N$, namely

$$
A_{c}=A_{0}+\frac{1}{N} \frac{\delta W^{(1)}}{\delta A} \circ \frac{\delta A}{\delta J}+\mathcal{O}\left(\frac{1}{N^{2}}\right)
$$

When no confusion with the integration variables in ( 3 ) exists, we shall drop the subscript " $c$ " from the c-number fields to simplify the notation.

The effective action functional may be defined in terms of the c-number fields ( 9 ) by a Legendre transformation in the usual way,

$$
\mathcal{S}[A, \psi, \bar{\psi}] \equiv W-J \circ A-\bar{\eta} \circ \psi-\bar{\psi} \circ \eta
$$

where $J, \bar{\eta}$ and $\eta$ are to be regarded as functionals of the mean fields by inverting eqs. (9). The result of this Legendre transformation is simply

$$
\mathcal{S}=-\bar{\psi} \circ G^{-1}[A] \circ \psi-\frac{1}{2} A \circ d^{-1} \circ A-i \operatorname{Tr} \ln G^{-1}[A]+\frac{i}{2 N} \operatorname{Tr} \ln D^{-1}[A]
$$

The photon inverse propagator in the last term is given by

$$
\begin{array}{r}
D^{-1}[A](x, y)^{\mu \nu}=\left(d^{-1}+\Pi[A]\right)(x, y)^{\mu \nu} \\
\Pi[A](x, y)^{\mu \nu} \equiv-i \operatorname{tr}\left\{\gamma^{\mu} G[A](x, y) \gamma^{\nu} G[A](y, x)\right\}
\end{array}
$$

where $\Pi$ is the polarization tensor in the presence of the potential $A$. The inverse propagator cannot be inverted without fixing a gauge, which may be done by a variety of standard methods.

The effective equations for the mean fields $\langle A\rangle \equiv<$ out $|A|$ in $>$ and $<\psi>\equiv<$ out $|\psi|$ in $>$ are given by

$$
\left.\frac{\delta \mathcal{S}}{\delta A(x)}\right|_{A=\langle A\rangle}=0 \quad ;\left.\quad \frac{\delta \mathcal{S}}{\delta \psi(x)}\right|_{\psi=\langle\psi\rangle}=0
$$


In the absence of sources the Dirac mean field vanishes. The integro-differential equations for the mean potential read

$$
\begin{aligned}
\partial_{\nu} F^{\mu \nu}(x) & =-i e^{2} \operatorname{tr}\left\{G[A](x, x) \gamma^{\mu}\right\}+\frac{i e^{2}}{2 N} \operatorname{Tr}\left\{D[A] \circ \frac{\delta \Pi[A]}{\delta A_{\mu}(x)}\right\} \\
& =-i e^{2} \operatorname{tr}\left\{G(x, x) \gamma^{\mu}\right\} \\
& +\frac{i e^{2}}{N} \int d^{4} x_{1} \int d^{4} x_{2} \operatorname{tr}\left\{\gamma^{\mu} G\left(x, x_{1}\right) \Sigma\left(x_{1}, x_{2}\right) G\left(x_{2}, x\right)\right\}
\end{aligned}
$$

where

$$
\Sigma\left(x_{1}, x_{2}\right) \equiv i \gamma^{\mu} G\left(x_{1}, x_{2}\right) \gamma^{\nu} D_{\nu \mu}\left(x_{2}, x_{1}\right)
$$

is the fermion self-energy. To leading order in $1 / N$ these are just the semi-classical Maxwell equations, obtained by replacing the electric current operator of the Dirac field by its expectation value. This leading order semi-classical equation already contains the dynamica! reaction of $e^{+} e^{-}$pairs created by a non-zero electric field (the Schwinger mechanism) back on the electric field itself [see J.M. Eisenberg lecture]. However, at leading order in $1 / N$ the created pairs can interact only through the mean field, not directly with each other. The order $1 / N$ term with the fermion selfenergy $\Sigma$ contains the quantum Compton scattering, bremmstrahlung and Coulomb interaction effects of these particles on each other and the backreaction of these effects on the self-consistent mean field. Clearly these processes are essential mechanisms for the approach to equilibrium and must be included in any realistic transport theory of a relativistic $e^{+} e^{-}$plasma. Higher order processes (such as multiple scattering) can be included by working to higher orders in the $1 / N$ expansion in a straightforward way.

Having derived the integro-differential equations for the mean fields in electrodynamics to order $1 / N$, we turn now to the Schwinger-Keldysh closed time path formulation of the effective action, in order to determine the correct combination of propagator functions needed to obtain a causal (and real) solution to these equations.

\section{THE SCHWINGER-KELDISH CLOSED TIME PATH FORMALISM}

The conventional path integral formalism used freely in the preceeding section defines transition elements between states at one time, $t$ (usually taken to be in the infinite past) to states at another time $t^{\prime}$ (in the distant future). If the class of paths is restricted to be the vacuum configuration at both of its endpoints, then the two states are the $\mid$ in $\rangle$ and $\langle o u t|$ vacuum states of scattering theory respectively. The 
generating functional $Z[J, \eta, \bar{\eta}]$ of Eq. ( 3 is the transition matrix element

$$
\left.Z[J, \eta, \bar{\eta}]\left(t, t^{\prime}\right)=\left\langle\text { out }, t^{\prime}\right| \text { in }, t\right\rangle_{J, \eta, \bar{\eta}}
$$

in the presence of the external source $J, \eta$ and $\bar{\eta}$.

By varying with respect to the external sources we obtain matrix elements of the Heisenberg field operators between the $|i n\rangle$ and $\langle o u t|$ states. For this reason we may refer to the conventional formulation of the generating functional $Z$ as the "in-out" formalism. The time-ordered Green's functions obtained in this way necessarily obey Feynman boundary conditions, and these are the appropriate ones for the calculation of transition probabilities and cross sections between the $|i n\rangle$ and $\langle$ out $|$ states. On the other hand the off-diagonal transition matrix elements of the in-out formalism are completely inappropriate if what we wish to consider is the time evolution of physical observables from a given initial condition. As we have remarked the in-out matrix elements are neither real, nor are their equations of motion causal at first order in $1 / N$, where direct self interactions between the fields appear for the first time. What we require is a generating functional for diagonal matrix elements of field operators with a corresponding modification of the Feynman boundary conditions on Green's functions to ensure causal time evolution. This "in-in" formalism was invented more than thirty years ago by Schwinger ${ }^{7}$ and later by Keldysh ${ }^{8}$, and is called the closed time path (CTP) method.

The basic idea of the CTP formalism is to take a diagonal matrix element of the system at a given time $t=0$ and insert a complete set of states into this matrix element at a different (later) time $t=t^{\prime}$. In this way one can express the original fixed time matrix element as a product of transition matrix elements from 0 to $t^{\prime}$ and the time reversed (complex conjugate) matrix element from $t^{\prime}$ to 0 . Since each term in this product is a transition matrix element of the usual (or time reversed) kind, standard path integral representations for each may be introduced. If the same external source operates in the forward evolution as the backward one, then the two matrix elements are precisely complex conjugates of each other, all dependence on the source drops out and nothing has been gained. However, if the forward time evolution takes place in the presence of one source $J_{+}$but the reversed time evolution takes place in the presence of a different source $J_{-}$, then the resulting functional is precisely the generating functional we seek. Indeed (setting $\eta=0, \bar{\eta}=0$ and $N=1$ here for simplicity),

$$
\begin{aligned}
& Z\left[J_{+}, J_{-}\right] \equiv \operatorname{Tr}\left\{\rho \overline{\mathcal{T}} \exp \left[-i \int_{0}^{t^{\prime}} d t d^{3} \vec{x} J_{-} A\right] \mathcal{T} \exp \left[i \int_{0}^{t^{\prime}} d t d^{3} \vec{x} J_{+} A\right]\right\} \\
= & \int[d \Phi]\left[d \Phi^{\prime}\right][d \Psi]\left\langle\Phi|\rho| \Phi^{\prime}\right\rangle
\end{aligned}
$$




$$
\begin{aligned}
& \times\left\langle\Phi^{\prime}\left|\overline{\mathcal{T}} \exp \left[-i \int_{0}^{t^{\prime}} d t d^{3} \vec{x} J_{-} A\right]\right| \Psi\right\rangle\left\langle\Psi\left|\mathcal{T} \exp \left[i \int_{0}^{t^{\prime}} d t d^{3} \vec{x} J_{+} A\right]\right| \Phi\right\rangle \\
= & \int[d \phi]\left[d \phi^{\prime}\right]\left\langle\phi|\rho| \phi^{\prime}\right\rangle \int[d \Psi] \int_{\phi}^{\Psi}\left[\mathcal{D} A_{+}\right]^{\prime}\left[\mathcal{D} \psi_{+}\right]\left[\mathcal{D} \bar{\psi}_{+}\right] \int_{\phi^{\prime}}^{\Psi}\left[\mathcal{D} A_{-}\right]^{\prime}\left[\mathcal{D} \psi_{-}\right]\left[\mathcal{D} \bar{\psi}_{-}\right] \times \\
& \exp \left[i \int_{0}^{\infty} d t d^{3} \vec{x}\left(L\left[A_{+}, \psi_{+}, \bar{\psi}_{+}\right]-L\left[A_{-}, \psi_{-}, \bar{\psi}_{-}\right]+J_{+} A_{+}-J_{-} A_{-}\right)\right] .
\end{aligned}
$$

where $\rho=|i n\rangle\langle i n|$ is the initial density matrix (an initial mean electric field corresponds to a coherent initial $|i n\rangle$ state), and where in the last equality a path integral representation for each transition element is introduced. Since the ime ordering in eq. ( 18 ) is forward (denoted by $\mathcal{T}$ ) along the time path from 0 tr $t^{\prime}$ in the second transition matrix element, but backward (denoted by $\overline{\mathcal{T}}$ ) along the same path from $t^{\prime}$ to 0 in the first matrix element, this generating functional receives the name of the closed time path generating functional.

The mean electromagnetic field expectation values are now given by,

$$
\left.\frac{\delta W_{\text {in }}\left[J_{+}, J_{-}\right]}{\delta J_{+}(x)}\right|_{J=0}=-\left.\frac{\delta W_{\text {in }}\left[J_{+}, J_{-}\right]}{\delta J_{-}(x)}\right|_{J=0}=\langle i n|A(x)| i n\rangle
$$

and the matrix connected two point functions are given by

$$
G^{a b}(x, y)=\left.\frac{\delta^{2} W}{\delta J_{a}(x) \delta J_{b}(y)}\right|_{J=0} ; \quad a=+,-,
$$

more explicitly

$$
\begin{aligned}
& G^{-+}(x, y) \equiv G_{>}(x, y)=i \operatorname{Tr}\{\rho \psi(x) \bar{\psi}(y)\}_{c o n} \\
& G^{+-}(x, y) \equiv G_{<}(x, y)=-i \operatorname{Tr}\{\rho \bar{\psi}(y) \psi(x)\}_{\text {con }} \\
& G^{++}(x, y)=i \operatorname{Tr}\{\rho \mathcal{T}[\psi(x) \bar{\psi}(y)]\}_{\text {con }}=\theta(x, y) G_{>}(x, y)+\theta(y, x) G_{<}(x, y) \\
& G^{--}(x, y)=i \operatorname{Tr}\{\rho \overline{\mathcal{T}}[\psi(x) \bar{\psi}(y)]\}_{\text {con }}=\theta(y, x) G_{>}(x, y)+\theta(x, y) G_{<}(x, y) .
\end{aligned}
$$

We may take over all the results of the previous section on the generating functional, effective action, and equations of motion of QED, provided only we substitute the CTP path ordered Green's function(s) for the ordinary Feynman propagators in internal lines, integrate over the full closed time contour, and satisfy the initial conditions at $t=0$ corresponding to the given density matrix $\rho$.

The causal equations of motion for nonequilibrium electrodynamics up to the next to leading order in $1 / N$ expansion take the form,

$$
\begin{aligned}
\partial_{\nu} F^{\mu \nu}(x)= & -\frac{i e^{2}}{2} \operatorname{tr}\left\{\left[G_{>}(x, x)+G_{<}(x, x)\right] \gamma^{\mu}\right\} \\
+ & \frac{2 e^{2}}{N} \mathcal{I} m \int_{0}^{t} d t_{1} d^{3} \vec{x}_{1} \int_{0}^{t_{1}} d t_{2} d^{3} \vec{x}_{2}\left\{\left[G_{>}\left(x_{1}, x\right)-G_{<}\left(x_{1}, x\right)\right] \gamma^{\mu} \times\right. \\
& {\left.\left[G_{>}\left(x, x_{2}\right) \Sigma_{>}\left(x_{1}, x_{2}\right)-G_{<}\left(x, x_{2}\right) \Sigma_{<}\left(x_{1}, x_{2}\right)\right]\right\} }
\end{aligned}
$$


The Wightman functions for the Dirac field satisfy

$$
\left(i \gamma^{\mu} \partial_{\mu}+\gamma^{\mu} A_{\mu}+i m\right) G_{>,<}(x, y)=0,
$$

together with the initial conditions implied by the first two members of eqs. (21), which satisfy the canonical equal time anticommutator condition,

$$
\left[G_{>}(x, y)-G_{<}(x, y)\right]_{t_{x}=t_{y}}=i \operatorname{Tr}\left\{\rho[\psi(x), \bar{\psi}(y)]_{+}\right\}_{t_{x}=t_{y}}=\delta^{3}(\vec{x}-\vec{y}),
$$

appropriate for Fermi-Dirac statistics.

For a complete initial value problem to order $1 / N$, one needs also the two-point function of the Maxwell field obtained by inverting (13) subject to some gauge condition. The simplest way to impose the gauge condition is to split it in the following way

$$
D_{\mu \nu}(x, y)=e^{2} d_{\mu \nu}(x, y)+\widetilde{D}_{\mu \nu}(x, y)
$$

with $d_{\mu \nu}$ the inverse of the differential operator ( 8 ) of the free action in a definite gauge. The gauge fixing can be performed at the levei of the free photon propagator once and for all, independently of the dynamical time evolution problem, and the non-trivial time evolution is contained entirely in $\widetilde{D}_{\mu \nu}$, which then obeys

$$
\begin{aligned}
\left(g^{\mu \lambda} \square-\partial^{\mu} \partial^{\lambda}\right) \widetilde{D}_{\lambda \nu}^{\lambda_{\lambda}<}(x, y) & =e^{2} \int_{0}^{t_{\Sigma}} d^{4} x_{1}\left[\Pi_{>}^{\mu \lambda}\left(x, x_{1}\right)-\Pi_{<}^{\mu \lambda}\left(x, x_{1}\right)\right] D_{\lambda \nu}\left(x_{1}, y\right)^{\rangle^{<}<} \\
& -e^{2} \int_{0}^{t_{y}} d^{4} x_{1} \Pi_{>,<}^{\mu \lambda}\left(x, x_{1}\right)\left[D_{\lambda \nu}\left(x_{1}, y\right)^{>}-D_{\lambda \nu}\left(x_{1}, y\right)^{<}\right] .
\end{aligned}
$$

A particularly useful gauge choice for practical implementation of the initial value problem on a computer is the Coulomb gauge, which has the advantage of clearly isolating the physical transverse modes of the photon and allowing the longitudinal and gauge modes to be eliminated from evolution problem, thereby making most efficient use of computer memory.

\section{BACK-REACTION IN BOOST INVARIANT COORDINATES}

Equipped with the above formalisms which are suitable for solving an initial value problem, I here present the resulting equations of motion for QED in the case where the initial conditions are symmetric under longitudinal boosts, and where only the leading order of the large $N$ expansion is taken into account. This symmetry is clearly displayed by introducing the light-cone variables $\tau$ and $\eta$

$$
z=\tau \sinh \eta \quad, \quad t=\tau \cosh \eta
$$


and by specifying the initial conditions of the fields at $\tau=\tau_{0}$, i.e., on a hyperbola of constant proper time.

Covariance of the theory guarantees that once the initial conditions have this symmetry, it will be conserved at any time. This allows us to expand the Dirac field in terms of Fourier modes at a fixed proper time $\tau$ :

$$
\psi(x)=\int \frac{d \mathbf{k}_{\perp} d k_{\eta}}{(2 \pi)^{3}} \sum_{s=1,2}\left[b_{\boldsymbol{\theta}}(\mathbf{k}) \psi_{\mathbf{k}_{\boldsymbol{A}}}^{+}(\tau) e^{i k_{\eta} \eta} e^{i \mathbf{k}_{\perp} \cdot \mathbf{x}_{\perp}}+d_{\mathbf{s}}^{\dagger}(-\mathbf{k}) \psi_{-\mathbf{k}_{\boldsymbol{\theta}}}^{-}(\tau) e^{-i k_{\eta} \eta} e^{-i \mathbf{k}_{\perp} \cdot \mathbf{x}_{\perp}}\right]
$$

If the electric field is in the $z$ direction and is a function of $\tau$ only, upon substituting eq. (28) into eq. (21) and using the boost invariant variables the system of equations (22)-(24) reduces, in the gauge $\left(A_{\tau}=0, A_{x}=0, A_{y}=0, A_{\eta}=A_{\eta}(\tau)\right)$, to

$$
\begin{gathered}
{\left[\gamma^{0}\left(\frac{d}{d \tau}+\frac{1}{2 \tau}\right)+i \gamma_{\perp} \cdot \mathbf{k}_{\perp}+i \gamma^{3} \pi_{\eta}+m\right] \psi_{\mathbf{k}_{\mathbf{s}}}^{ \pm}(\tau)=0,} \\
\frac{1}{\tau} \frac{d E(\tau)}{d \tau}=-\frac{e}{2 \tau}\left\langle i n\left|\left[\psi^{\dagger}, \gamma^{0} \gamma^{3} \psi\right]\right| i n\right\rangle,
\end{gathered}
$$

where $\pi_{\eta} \equiv\left(k_{\eta}-e A_{\eta}\right) / \tau$.

For an initial coherent state which satisfies $b_{s}(\mathbf{k})|i n\rangle=d_{\bullet}(-\mathbf{k})|i n\rangle=0$ (an adiabatic vacuum) the Maxwell eq. (30) reads

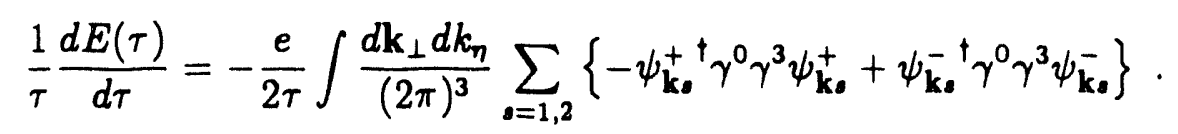

The renormalization of the above equation can be done by the adiabatic regularization technique. Unfortunately, it is not applicable for renormalization beyond the leading order of the $1 / N$ expansion. However, recently we found a simple prescription for removing the logarithmic divergences by recognizing that renormalization group invariant quantities like $e E$ do not depend on the momentum cutoff $\Lambda$, as long as we rescale the coupling constant according to the standard renormalization group flow to the given order in $1 / N$. For a fixed renormalized coupling constant $e_{R}$ and an electric field $E_{R}$, the corresponding bare quantities in the lowest order in the large $N$ expansion are defined via

$$
\begin{array}{r}
e_{R}^{2} \equiv Z_{\Lambda} e^{2} ; \quad E_{R} \equiv Z_{\Lambda}^{-1 / 2} E \quad ; \quad e E=e_{R} E_{R} \\
Z_{\Lambda}=\left[1+e^{2} \delta e^{2}(\Lambda)\right]^{-1}=\left[1-e_{R}^{2} \delta e^{2}(\Lambda)\right] \\
\delta e^{2}(\Lambda)=\frac{1}{4 \pi^{2}} \int_{0}^{\Lambda} d k\left[\frac{k^{2}}{\left(k^{2}+m^{2}\right)^{\frac{3}{2}}}-\frac{k^{4}}{3\left(k^{2}+m^{2}\right)^{\frac{5}{2}}}\right]
\end{array}
$$

Using the first line of eq. (32) allows us to rewrite eq. (31) in the form

$$
\frac{e_{R}}{\tau} \frac{d E_{R}(\tau)}{d \tau}=-\frac{e_{R}^{2} / Z_{\Lambda}}{2 \tau} \int^{\Lambda} \frac{d \mathbf{k}_{\perp} d k_{\eta}}{(2 \pi)^{3}} \sum_{:=1,2}\left\{-\psi_{\mathbf{k}_{\ell}}^{+}{ }^{\dagger} \gamma^{0} \gamma^{3} \psi_{\mathbf{k}_{\ell}}^{+}+\psi_{\mathbf{k}_{\ell}}^{-}{ }^{\dagger} \gamma^{0} \gamma^{3} \psi_{\mathbf{k}_{\ell}}^{-}\right\}
$$



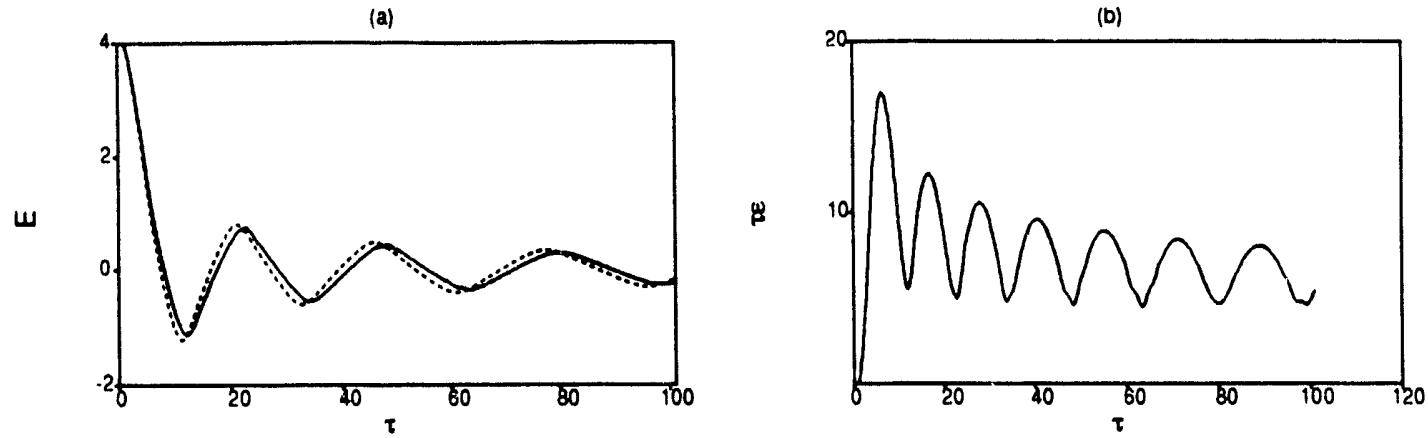

Figure 1: Proper time evolution of (a) the rescaled electric field $E(\tau) \rightarrow e E / \mathrm{m}^{2}$, and (b) the energy density of the fermion field $\epsilon$ multiplied by $\tau$, for initial conditions at $\tau=1$ ith a rescaled initial electric field $E(\tau=1)=4$ and $e^{2} / m^{2}=1$.

which is a renormalization group invariant, and therefore the right hand side is cutoff independent. This cutoff independence has been checked both for scalar QED in flat coordinates and boost invariant coordinates.

Figures 1-2 summarize the results of the numerical simulation for the evolution of the system (29)-(30) in $1+1$ dimensions. In Fig. 1(a) the proper time evolution of the electric field is presented, where the initial conditions where fixed at $\tau=1$. The amplitude falls off as a result of the particle production and the expansion of the system. Having the solution for the fermion mode functions, we calculated the expectation value of the energy-momentum tensor. Fig. 1(b) shows the fermion part of the $T_{\tau \tau}$ component, which can be identified with the energy density $\epsilon$ in the comoving frame.

If we expand the field $\psi$ in terms of the time-dependent creation and annihilation operators of the lowest-order adiabatic vacuum

$$
\psi(x)=\int \frac{d k_{\eta}}{2 \pi}\left[a\left(k_{\eta} ; \tau\right) y_{k_{\eta}}^{+}(\tau) e^{i k_{\eta} \eta}+c^{\dagger}\left(-k_{\eta} ; \tau\right) y_{-k_{\eta}}^{-}(\tau) e^{-i k_{\eta} \eta}\right]
$$

where $y_{k_{m}}^{ \pm}$are the lowest order adiabatic mode functions, we can relate these operators to those in eq. (28) via a Bogolyubov transformation

$$
a\left(k_{\eta} ; \tau\right)=\alpha\left(k_{\eta} ; \tau\right) b\left(k_{\eta}\right)+\beta^{*}\left(k_{\eta} ; \tau\right) d^{\dagger}\left(-k_{\eta}\right)
$$

where $\alpha$ and $\beta$ are functions of the known mode functions $\psi_{k_{n}}^{ \pm}$and $y_{k_{n}}^{ \pm}$. Due to the boost invariant symmetry the rapidity distribution $d N / d y$ is equal to the particle density $d N / d \eta$ which is given by

$$
d N / d \eta=\int \frac{d k_{\eta}}{2 \pi}\left\langle a^{\dagger}\left(k_{\eta} ; \tau\right) a\left(k_{\eta} ; \tau\right)+c^{\dagger}\left(-k_{\eta} ; \tau\right) c\left(-k_{\eta} ; \tau\right)\right\rangle .
$$



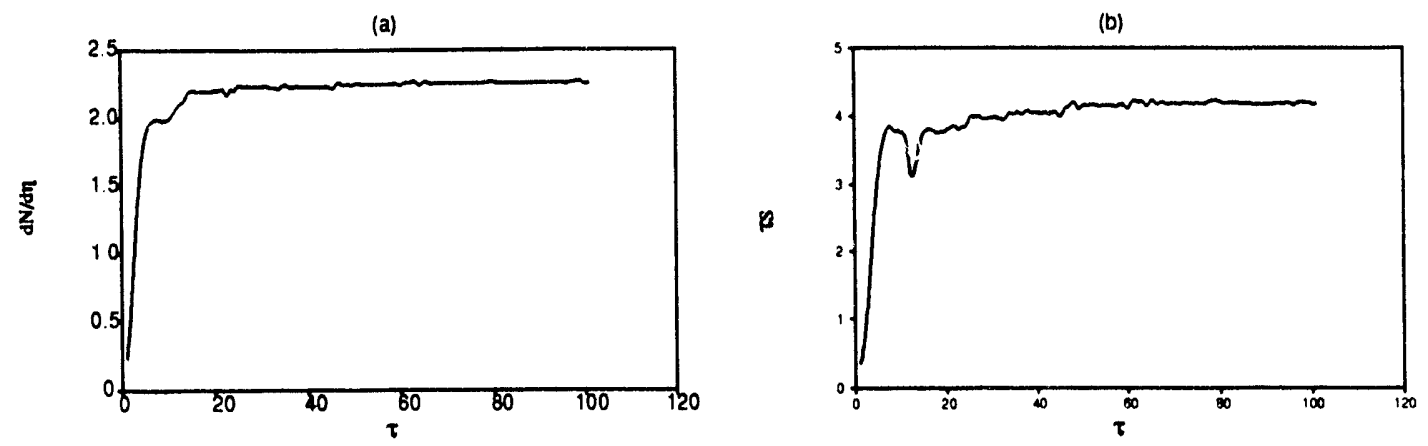

Figure 2: Proper time evolution of (a) $d N / d \eta$, and (b) Boltzmann entropy density multiplied by $\tau$.

As is shown in Fig. 2(a), most of the production takes place in the early stages. Identifying this particle density with a Boltzmann-like phase density $\tilde{f}$, in Fig. 2(b) we plot the Boltzmann entropy density in the comoving frame, which is defined as

$$
s(\tau)=-\frac{1}{\tau} \int \frac{d k_{\eta}}{2 \pi}\{\tilde{f} \ln \tilde{f}+(1-\tilde{f}) \ln (1-\tilde{f})\} .
$$

By the time particle production has nearly ceased, the quantity $\tau s$ is roughly a constant, as expected in a hydrodynamics treatment with no source term of particle production $^{24}$.

Finally, as is shown in J.M. Eisenberg's lecture at this school, a comparison of the field theory results with those of a transport equation with a Schwinger source term for particle production shows similar results for the case of an homogeneous electric field. In the curvilinear coordinates the transport Boltzmann-Vlassov equation reads

$$
\begin{aligned}
& \frac{\partial f}{\partial \tau}+e \tau E(\tau) \frac{\partial f}{\partial p_{\eta}}=-\left[1-2 f\left(p_{\eta}, \tau\right)\right] e \tau|E(\tau)| \times \ln \left[1-\exp \left(-\frac{\pi m^{2}}{e|E(\tau)|}\right)\right] \delta\left(p_{\eta}\right), \\
& -\tau \frac{d E}{d \tau}=j_{\eta}=j_{\eta}^{\text {cond }} \leftarrow j_{\eta}^{\text {pol }} \\
& j_{\eta}^{\text {cond }}=2 e \int \frac{d p_{\eta}}{2 \pi \tau p_{\tau}} p_{\eta} f\left(p_{\eta}, \tau\right) \\
& j_{\eta}^{\text {pol }}=-\left[1-2 f\left(p_{\eta}=0, \tau\right)\right] \frac{m e \tau}{\pi} \operatorname{sign}[E(\tau)] \ln \left[1-\exp \left(-\frac{\pi m^{2}}{|e E(\tau)|}\right)\right] .
\end{aligned}
$$

In Fig. 1(a) we see that the results of the Boltzmann-Vlassov equation (dashed curve) agree very well with those of the mean field calculation (solid curve) as in the case of flat coordinates with an homogenous electric field.

The agreement of the leading order of the large $N$ expansion and the kinetic approach is encouraging since the latter approach is much simpler for numerical simulations. In the next order, one needs to make a gradient expansion of the field theory equations to obtain a transport theory ${ }^{25}$. It is necessary to see whether the 
truncation of the gradient expansion reasonably approximates the full field theory results after they are coarse grained in the momentum space. If the next to leading order calculations will reproduce similar results in the homogeneous case, one would then be more confident that the kinetic approach is appropriate for describing more realistic situations (inhomogeneity and higher order collision terms which can be easily derived in the large $N$ expansion). Using the field theory approach, these more difficult problems are beyond the scope of present computing capability.

The CTP project I presented here was done in collaboration with Fred Cooper, Emil Mottola, Juan Pablo Paz, Salman Habib, and Paul Anderson. The last part I presented here was done in collaboration with Fred Cooper, Emil Mottola, Judah Eisenberg, and Ben Svetitsky.

\section{References}

[1] A. Bialas and W. Czyż, Phys. Rev. D 30, 2371 (1984); ibid. 31, 198 (1985); Z. Phys. C28, 255 (1985); Nucl. Phys. B267, 242 (1985); Acta Phys. Pol. B 17, 635 (1986).

[2] A. Bialas, W. Czyż, A. Dyrek, and W. Florkowski, Nucl. Phys. B296, 611 (1988).

[3] K. Kajantie and T. Matsui, Phys. Lett. 164B, 373 (1985).

[4] G. Gatoff, A. K. Kerman, and T. Matsui, Phys. Rev. D 36, 114 (1987).

[5] K. Geiger and B. Muller, Nucl.Phys. B369, 600 (1992).

[6] X. -N. Wang and M. Gyulassy, Phys. Rev. D 41, 3501 (1992).

[7] J. Schwinger, J. Math. Phys. 2, 407 (1961).

[8] K. T. Mahanthappa, Phys. Rev. 126, 329 (1962).

[9] L. V. Keldysh, JETP 20, 1018 (1965).

[10] K. Wilson, Phys. Rev. D 7, 2911 (1973).

[11] S. Coleman, R. Jackiw, and H. D. Politzer, Phys. Rev. D 10, 2491 (1974).

[12] J. Cornwall, R. Jackiw, and E. Tomboulis, Phys. Rev. D 10, 2428 (1974).

[13] C. M. .Bender, F. Cooper, and G. S. Guralnik, Ann. Phys. 109, 165 (1977); R. Root, Phys. Rev. D 11, 831 (1975). 
[14] H. -Th. Elze, M. Gyulassy and D. Vasak, Nucl. Phys. B276, 706 (1986).

[15] C. Best and J. M. Eisenberg, Phys. Rev. D 47,4639 (1993).

[16] F. Cooper and E. Mottola, Phys. Rev. D 40, 456 (1989).

[17] Y. Kluger, J. M. Eisenberg, B. Svetitsky, F. Cooper, and E. Mottola, Phys. Rev. Lett. 67, 2427 (1991).

[18] Y. Kluger, J. M. Eisenberg, B. Svetitsky, F. Cooper, and E. Mottola, Phys. Rev. D 45, 4659 (1992).

[19] F. Cooper, in Particle Production in Highly-Excited Matter, Proc. NATO Advanced Study Institute, Il Ciocco, Italy, July, 1992, edited by H. Gutbrod and J. Rafelsk:.

[20] P. Anderson, F. Cooper, S. Habib, Y. Kluger, E. Mottola, and J. P. Paz, in preparation.

[21] F. Cooper, J. M. Eisenberg, Y. Kluger, E. Mottola, and B. Svetitsky, Phys. Rev. D 48, 190 (1993).

[22] J. Schwinger, Phys. Rev. 82, 664 (1951).

[23] J. D. Bjorken, Phys. Rev. D27, 140 (1983).

[24] F. Cooper, G. Frye and E. Schonberg, Phys. Rev. D11, 192 (1975).

[25] E. Calzetta and B. L. Hu, Phys. Rev. D 37, 2878 (1988). 

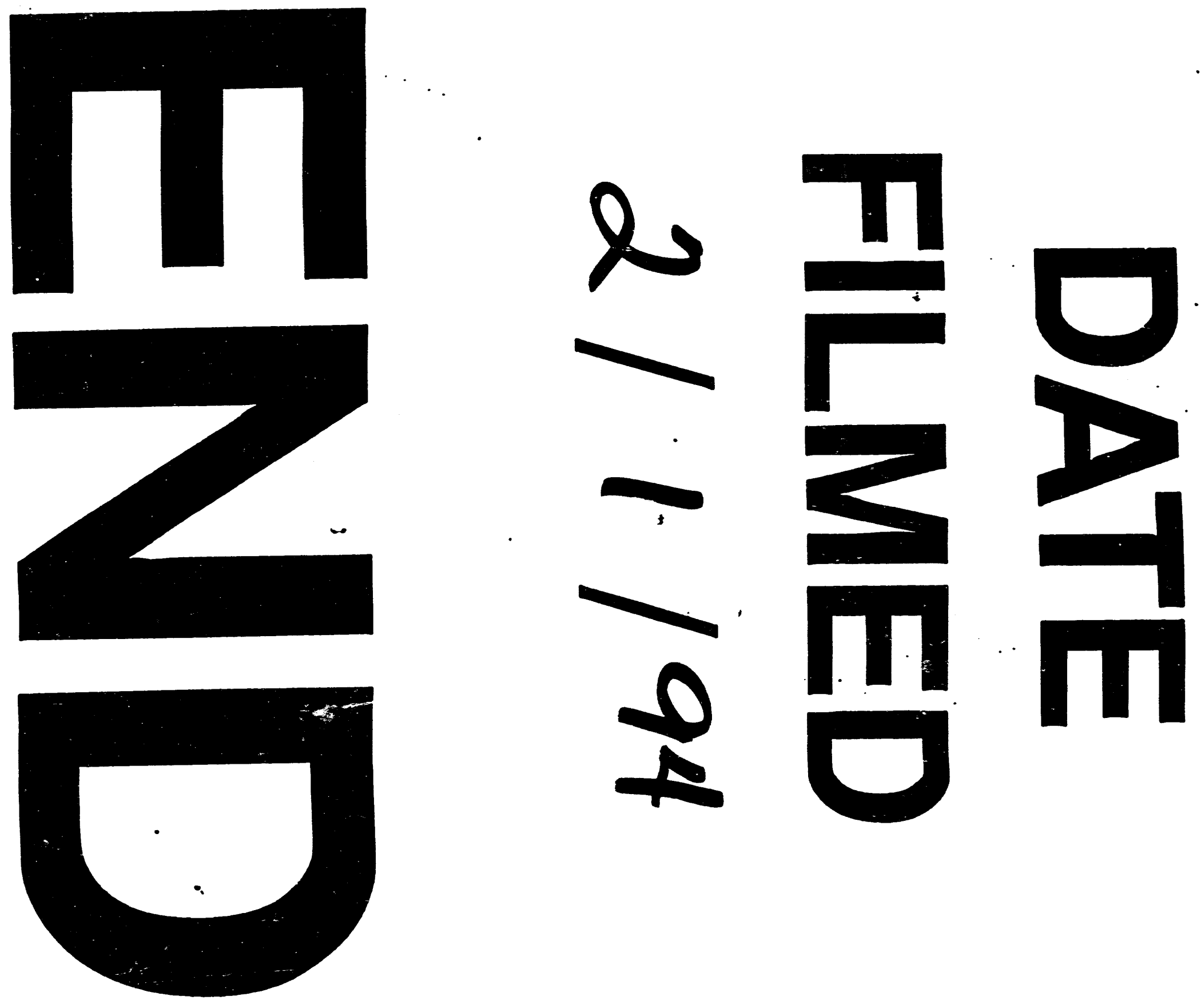


$$
\text { - }
$$

(2)

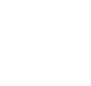

- 